\title{
Editorials
}

\section{Diagnosis of coeliac disease in children in primary care and clinical implications}

\section{HISTORICAL APPROACH TO DIAGNOSIS}

Since the introduction of the Crosby capsule la device used for obtaining biopsies of small bowel mucosa, necessary for the diagnosis of various small bowel diseases) in the 1970s, small intestinal biopsy has been the cornerstone of diagnosis of coeliac disease. In paediatric practice, successive position statements from the European Society of Paediatric Gastroenterology, Hepatology and Nutrition (ESPGHAN) have defined criteria for childhood diagnosis: initially based on three successive biopsies at presentation, on a gluten-free diet, and then challenge. Since 1990, the diagnosis has been dependent on a single biopsy with a clear clinical response to a gluten-free diet, supported by positive serology. ${ }^{1}$ This approach is similar to that recommended for adults ${ }^{2}$ and has been adopted by the National Institute for Health and Care Excellence (NICE). ${ }^{3}$

\section{ADVANCES IN UNDERSTANDING}

Great strides have been made in serological assessment and understanding coeliac disease pathogenesis since the 1990 guidelines were introduced. First, the nonspecific gliadin antibodies used at that time were supplanted by the highly specific IgA endomyseal antibody (EMA) test. Subsequently tissue transglutaminase-2 (tTG) was identified as the true autoantigen causing EMA reactivity. EMA testing depends on immunofluorescence, giving a yes-no answer dependent on operator expertise. Importantly, assay of tTG autoantibody is performed by enzyme-linked immunosorbent assay (ELISA), allowing a quantitative assessment of immune response. The second major diagnostic advance was the determination of the relevant human leukocyte antigen (HLA) types that allow presentation of gliadin/ tTG complexes to $T$ cells, thus causing the autoimmune response to tTG. Persons of tissue types HLA-DQ2 and HLA-DQ8 (more than a quarter of the UK population) make up $99 \%$ of cases of coeliac disease, and true coeliac disease is very unusual in someone who is not DQ2 or DQ8 positive. ${ }^{4}$

\section{COELIAC DISEASE: A COMMON DISORDER}

The widespread introduction of EMA and TTG testing for coeliac disease led to recognition that the disease is much more common than previously thought; affecting about $1 \%$ of the population. There has therefore been a

.even now around $80 \%$ of cases remain unrecognised in both adults and children.

shift towards recognition of people with more subtle symptoms, but even now around $80 \%$ of cases remain unrecognised in both adults and children compared with what would be expected in a screening programme. ${ }^{5}$ The long-term consequences of truly asymptomatic coeliac disease are unknown, and, as such, population screening is not yet indicated. However, the challenge is to identify people with active coeliac disease but relatively subtle symptoms. Symptoms that should prompt screening for coeliac disease include persistent loose stools, faltering growth, unexplained short stature, abdominal pain, vomiting, abdominal distension, chronic constipation, dermatitis herpetiformis, dental enamel defects, osteoporosis or pathological fractures, delayed menarche, unexplained anaemia, iron-deficient anaemia unresponsive to treatment, recurrent aphthous stomatitis, unexplained liver disease, and lassitude or weakness.

\section{CAN COELIAC DISEASE BE DIAGNOSED SECURELY WITHOUT BIOPSY IN CHILDREN?}

In comparison with adult practice, there are potential difficulties in delivering appropriately swift diagnostic endoscopy for coeliac disease. It is now considered appropriate to use general anaesthesia rather than sedation for paediatric endoscopy, particularly in young patients. There are substantially fewer paediatric gastroenterologists than adult specialists, in the UK and across Europe. There has also been an increase in demand for paediatric endoscopy, not only due to the increase in recognition of children with coeliac disease, ${ }^{5}$ but also to increasing numbers of children requiring endoscopy for inflammatory bowel disease and emerging disorders such as eosinophilic oesophagitis. Therefore publication of studies that demonstrated that a high titre of $\lg \mathrm{A}$ tTG antibodies accurately predicted the finding of diagnostic histological changes on biopsywere important. 6.7 These prompted a systematic review of the available data by the coeliac working group of ESPGHAN, and publication in 2012 of revised diagnostic criteria for coeliac disease in children. 89 These revised criteria included the potential for diagnosis of coeliac disease without biopsy - but with a strict protocol of blood testing, in children with clear symptoms, presenting with a high titre of $\mathrm{tTG}$ autoantibodies (more than 10 times the upper limit of normal), in whom a second blood test confirmed both a positive endomyseal antibody and that the patient was HLA-DQ2 or HLA-DQ8. In all other cases a biopsy would be required as previously. These guidelines have been reviewed by the coeliac working group of the British Society of Paediatric Gastroenterology, Hepatology and Nutrition (BSPGHAN) together with Coeliac UK, who have jointly published recommendations for coeliac disease diagnosis and management for UK paediatric practice. ${ }^{10}$ This does not encompass non-coeliac wheat intolerance.

\section{RECOMMENDATIONS BY BSPGHAN AND COELIAC UK}

BSPGHAN and Coeliac UK recommend that all children with suspected coeliac disease should have their diagnosis established by a paediatric gastroenterologist, and their follow-up under the care of a paediatric gastroenterologist or a paediatrician with a special interest in coeliac disease, with access to appropriately skilled paediatric dietetic services. There should be a low threshold for testing coeliac serology in both symptomatic children (see above) and those with associated conditions, as so many cases remain undiagnosed.

\section{SYMPTOMATIC CHILDREN WITH POSITIVE tTG TESTING AT HIGH TITRE (X 10x ULN)}

Either a small bowel biopsy may be performed as previously, or a second blood test taken for $\lg A$ EMA and HLA testing. If IgA EMA positive and the child is confirmed to be HLA$\mathrm{DQ} 2$ or HLA-DQ8, then the diagnosis may be confirmed without biopsy.

\section{Important practice points:}

1. A single positive $T T G$ or EMA blood test is insufficient for diagnosis. 


\section{"All children with suspected coeliac disease should have their diagnosis established by a paediatric gastroenterologist.}

2. A gluten-free diet should not be started before the diagnosis is confirmed, even if the $\mathrm{tTG}$ antibody titre is high.

If $\mathrm{tTG}$ is raised at more modest levels a biopsy will be required. For children who are IgA deficient, a biopsy will also still be required as IgG tTG antibodies do not reliably predict histology.

\section{ASYMPTOMATIC CHILDREN WITH ASSOCIATED CONDITIONS}

For asymptomatic children who have associated conditions (for example, type 1 diabetes, IgA deficiency, Down's syndrome, Williams or Turner syndromes, autoimmune thyroiditis, autoimmune liver disease, unexplained raised transaminases) or who are relatives of a coeliac patient, there should be a low threshold to consider coeliac disease. Certainly the development of symptoms or finding of iron deficiency or anaemia should prompt testing of tTG antibodies. Initial testing of HLA type in at-risk groups may allow long-term exclusion of the diagnosis (that is, the child is not DQ2 or DQ8). Such testing is likely to be carried out in secondary care. For the child who is DQ2 or DQ8 positive but has negative tTG screening, there is no indication for further testing for 3 years unless the child becomes symptomatic. For the child found to be tTG positive, referral to a paediatric gastroenterologist for consideration of biopsy is indicated. The decision about biopsy would depend on the titre of tTG antibody.

\section{DIFFERENCES BETWEEN ADULT AND PAEDIATRIC GASTROENTEROLOGY PRACTICE}

For the moment, the British Society of Gastroenterology is maintaining a biopsybased diagnostic approach for adults with suspected coeliac disease. Thus, there are potential differences between adult and paediatric diagnostic approaches. Potential advantages of the new approach for symptomatic children include rapidity of diagnosis las mentioned previously, there are substantially fewer paediatric than adult gastroenterologists across the UK, and paediatric endoscopy usually requires general anaesthesial and avoiding an invasive procedure that children may find unpleasant. Potential concerns about this approach include the variability between tTG assay systems in use (there are now more than 20 commercial competitors, some of which show different performance characteristics), which may make a precise cut-off indicating enteropathy less certain than previous studies have suggested. ${ }^{11}$ Conversely, it is also known that artefacts of biopsy handling and cutting could give significant unreliability in diagnostic histological interpretation ${ }^{12}$ and therefore it is possible that no universal gold standard exists. In adults, endoscopy is usually rapidly available and better tolerated, and gastroenterologists may perform followup endoscopy to determine histological improvement. The Coeliac Disease Guideline Development Group of NICE is currently reviewing the evidence base, with a view to updating NICE guidelines within the next 2 years. However, children diagnosed by the new ESPGHAN/BSPGHAN criteria should be viewed as having a secure diagnosis and commenced on a gluten-free diet.

Within primary care, identification of previously unrecognised childhood cases may reduce overall use of health care services, although costs may rise through tests and referral with prescription of a gluten-free diet. The higher costs of paediatric endoscopy under general anaesthetic compared with adult sedation-based procedures should provide cost savings for a blood test-based diagnostic protocol. However, both cost analysis and in-use validation of the new guidelines will be needed. Importantly, there is an ongoing pan-European study in children to examine the impact in practice of the new guidelines on diagnostic security.

\section{Simon Murch,}

Professor of Paediatrics, Warwick Medical School, Coventry (on behalf of the Coeliac Disease Working Group of BSPGHAN].

\section{Richard Stevens,}

GP, East Oxford Health Centre, Cowley, Oxford.

\section{Sarah Sleet,}

Chief Executive, Coeliac UK, High Wycombe.

\section{Provenance}

Commissioned; not externally peer reviewed.

DOI: 10.3399/bjgp14X680785

\section{ADDRESS FOR CORRESPONDENCE}

Simon Murch

Division of Metabolic and Vascular Health, Warwick Medical School, Clinical Sciences Building, Clifford Bridge Road, Coventry, CV2 2DX, UK.

E-mail: s.murchawarwick.ac.uk

\section{REFERENCES}

1. Revised criteria for diagnosis of coeliac disease. Report of working group of European Society of Paediatric Gastroenterology and Nutrition. Arch Dis Child 1990; 65(8): 909911

2. British Society for Gastroenterology. BSG guidance on coeliac disease 2010. The management of adults with coeliac disease. www.bsg.org.uk/sections/small-bowelnutrition-articles/bsg-guidance-on-coeliacdisease-2010.html (accessed 7 Jul 2014).

3. National Institute of Health and Care Excellence. Coeliac disease: recognition and assessment of coeliac disease. London: NICE, 2009. http://publications.nice.org.uk/coeliacdisease-cg86 (accessed 7 Jul 2014).

4. Abadie V, Sollid LM, Barreiro LB, Jabri B. Integration of genetic and immunological insights into a model of celiac disease pathogenesis. Annu Rev Immunol 2011; 29: 493-525.

5. Whyte LA, Jenkins HR. The epidemiology of coeliac disease in South Wales: a 28-year perspective. Arch Dis Child 2013; 98(6): 405-407.

6. Hill PG. Holmes GK. Coeliac disease: a biopsy is not always necessary for diagnosis. Aliment Pharmacol Ther 2008; 27(7): 572-577.

7. Dahlbom I, Korponay-Szabó IR, Kovács JB, et al. Prediction of clinical and mucosal severity of coeliac disease and dermatitis herpetiformis by quantification of $\lg A / \lg G$ serum antibodies to tissue transglutaminase. $J$ Pediatr Gastroenterol Nutr 2010; 50(2): 140-146.

8. Giersiepen K, Lelgemann M, Stuhldreher N et al. Accuracy of diagnostic antibody tests for coeliac disease in children: summary of an evidence report. J Pediatr Gastroenterol Nutr 2012; 54(2): 229-241.

9. Husby S, Koletzko S, Korponay-Szabó IR, et al. European Society for Pediatric Gastroenterology, Hepatology, and Nutrition guidelines for the diagnosis of coeliac disease. J Pediatr Gastroenterol Nutr 2012; 54(1): 136-160.

10. Murch $S$, Jenkins $H$, Auth $M$, et al. Joint BSPGHAN and Coeliac UK guidelines for the diagnosis and management of coeliac disease in children. Arch Dis Child 2013; 98(10): 806-811.

11. Beltran L, Koenig M, Egner W, et al. Hightitre circulating tissue transglutaminase-2 antibodies predict small bowel villous atrophy, but decision cut-off limits must be locally validated. Clin Exp Immunol 2014; 176(2): 190-198

12. Taavela J, Koskinen O, Huhtala $\mathrm{H}$, et al. Validation of morphometric analyses of small-intestinal biopsy readouts in celiac disease. PLoS One 2013; 8(10): e76163. 